\title{
Rectal Colonization with Acinetobacter baumannif and the Risk of Blood stream Infection in Neonates
}

S. Khurana' ${ }^{1}$ S.S. Saini' ${ }^{1}$, H. Kaur², M. Biswal2 ${ }^{2}$, P. Kumar ${ }^{1}$

${ }^{1}$ Department of Pediatrics, ${ }^{2}$ Department of Medical Microbiology, Post Graduate Institute of Medical Education and Research,, Chandigarh, India.

\section{* INTRODUCTION}

- Acinetobacter is a ubiqitous organism found in hospitals and is known to spread by fomite based transmission from contaminated hand of health care workers and unclean equipments

- Acinetobacter has emerged as an important nosocomial pathogen in last decade

- Identified as the most common isolate causing neonatal sepsis ${ }^{1}$ in developing countries

- Important cause of health care associated infection

- Majority of hospital strains are drug resistant

- Neonates admitted in NICU are particularly prone to multi-drug resistant isolates of A.baumannii ${ }^{2}$

- Gastrointestinal colonization may be an important risk factor for acinetobacter bacteremia $^{3}$, but literature for same in neonates is lacking

\section{* oBjECTIVES}

To determine association between Rectal colonization with Acinetobacter baumanil and subsequent development of bacteremia with Acinetobacter among neonates admitted in NICU

\section{* MATERIAL and METHOds}

- Setting: Level-III NICU, Post Graduate Institute of Medical Education and Research, Chandigarh

- Duration: Aug 2015 to Jan 2016

- Design: Prospective observational study

- Ethical approval: The study was approved

by Institute's Ethical Committee

Participants: All neonates admitted in NICU were recruited

\section{METHODS (CONTD...)}

Protocol

- Rectal swabs were obtained from all the neonates admitted in NICU. The rectal swabs were repeated at weekly interval

- The swabs were inoculated onto blood and MacConkey agar within two hours of collection

- Identification of $A B$ was done by MALDITOF

- Antibiotic susceptibility was carried out by Kirby Bauer disc diffusion test

- The neonates were followed up for development of BSI during their NICU stay

- BSI data: Collected from existing surveillance system of the unit, using standard definitions

* RESUlts

- Total Neonates screened over the study period $=155$

- Number of rectal swabs sent over the study period $=250$

- Number of rectal swabs positive for Acinetobactor bauminnii= 24 in 19 neonates

- Out of the 19 neonates with gutcolonization, $4(21 \%)$ developed BSI due to $A B$

- Among the non-colonized babies $(n=136)$, eight $(5.8 \%)$ developed $\mathrm{BSI}$ due to $\mathrm{A}$ baumannii.

- The gut colonization by Acinetobactor baumannii was associated with $\mathrm{A}$ baumannii -BSI [RR $3.57(95 \% \mathrm{Cl} 1.00$ 13.03), p-value 0.05)
Table 1: Baseline characteristics \& Study findings

\begin{tabular}{|l|c|}
\hline Characteristics & Values \\
\hline Mean Gest age (wks) & $30.6 \pm 3.4$ \\
\hline Mean Birth weight (gms) & $1304 \pm 603$ \\
\hline Median post natal age (days) & $10 \pm 16.09$ \\
\hline $\begin{array}{l}\text { Median PNA at positive swab } \\
\text { (days) }\end{array}$ & $6.5 \pm 4.4$ \\
\hline $\begin{array}{l}\text { Neonates with repeat swab } \\
\text { negative (\%) }\end{array}$ & $6(4.4)$ \\
\hline $\begin{array}{l}\text { Median age to clearance } \\
\text { (days) }\end{array}$ & $7.5 \pm 3.8$ \\
\hline
\end{tabular}

* CONClusions

- Rectal Colonization with Acinetobacter baumanii has a borderline significant association with Acinetobacter BSI

- Rectal colonisation might help to predict subsequent bacteremia with acinetobacter baumannii

- Vigilant monitoring of colonised neonates and their cohorting can decrease Acinectobacter sepsis.

* References

1. Investigators of Delhi Neonatal Infection Study (DeNIS) collaboration. Lancet Global health 2016;04:e752-60

2. Berezin E, Guillou M, Towner J. Microbiology, Epidemiology, Infections, Management. 1995:109-12.

3. Marchaim D, Venezia S, Schwartz D, et al. J of Clinical Microbiology. May 2007;45:1551-55.

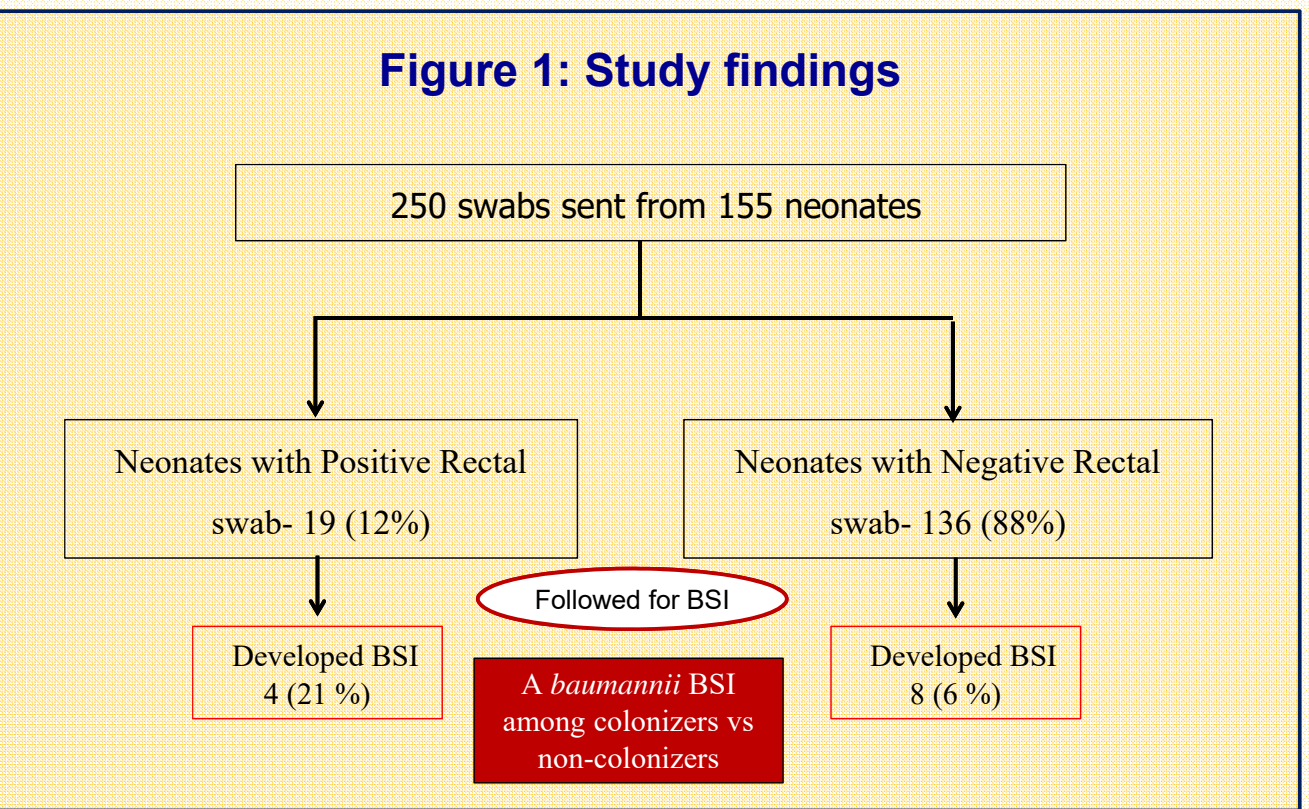

